\title{
Effect of variations in the gas mixture compositions on the timing and charge of glass RPC
}

\author{
A. K. Sikdar, ${ }^{a}$ J. Sadiq, ${ }^{a, b, 1}$ and P. K. Behera ${ }^{a}$ \\ ${ }^{a}$ Physics Department, Indian Institute of Technology Madras, Chennai, India \\ ${ }^{b}$ Physics Department, Islamic University of Science and Technology, Kashmir, India \\ E-mail: sadiqvengad@gmail.com
}

\begin{abstract}
The India-based Neutrino Observatory (INO) is a project aimed at building a large underground laboratory to explore the Earth's mater effects on the atmospheric neutrinos in multi$\mathrm{GeV}$ range. INO will host a 50 kton magnetized iron calorimeter detector (ICAL) in which Resistive Plate Chambers(RPCs) will be the active detector elements. In ICAL, 28,800 glass RPCs of $2 \mathrm{~m} \times$ $2 \mathrm{~m}$ size will be operated in the avalanche mode. A small variation in the compositions of ionizing gaseous medium in the RPC affects its performance. Study of the charge distribution of the RPC at different gas compositions is necessary to optimize the gas mixture.

An RPC made with glass plates of dimension $30 \mathrm{~cm} \times 30 \mathrm{~cm}$ was operated in avalanche mode with a gas mixture of $\mathrm{C}_{2} \mathrm{H}_{2} \mathrm{~F}_{4} / i \mathrm{C}_{4} \mathrm{H}_{10} / \mathrm{SF}_{6}$. We have studied the performance of these RPCs at the same ambient conditions. The percentages of the $i C_{4} H_{10}$ or $S F_{6}$ were varied and its effect on the performance of RPC were studied. The study of the charge distribution and time resolution of the RPC signals at different gas compositions is presented in this paper.
\end{abstract}

Keywords: Neutrino detectors; Resistive-plate chambers; Detector design and construction technologies and materials

\footnotetext{
${ }^{1}$ Corresponding author.
} 


\section{Contents}

1 Introduction 1

2 Experimental setup 2

2.1 Calibration of the gas system 2

3 Performance of RPC at different percentages of $\mathbf{S F}_{6} 3$

3.1 Charge distribution 3

3.2 Time resolution 4

4 Performance of RPC at different percentages of Isobutane $\left(\mathrm{iC}_{4} \mathrm{H}_{10}\right)$

4.1 Charge distribution 5

4.2 Time resolution 5

5 Conclusion 5

\section{Introduction}

Resistive Plate Chambers (RPCs) have found its applications in the particle physics experiments as well as in medical fields [1,2]. Most of the high energy experiments prefer the RPCs as tracking detectors because of their excellent position and time resolutions [3]. The India-based Neutrino Observatory (INO) has planned to build an underground laboratory which will house a $50 \mathrm{kton}$ magnetised Iron CALorimeter (ICAL) detector [4]. The ICAL detector aims to determine the neutrino mass hierarchy and to improve the precision bounds on $\theta_{23}$ and $\Delta m_{32}^{2}$ from the Earth's mater effects on the atmospheric $v_{\mu}$ and $\bar{v}_{\mu}$ propagation. The ICAL will consist of 151 horizontal layers of $5.6 \mathrm{~cm}$ thick iron plates interleaved by $4 \mathrm{~cm}$ air gaps in which the detector elements will be placed. The Resistive Plate Chambers (RPCs) of dimension $2 \mathrm{~m} \times 2 \mathrm{~m}$ are chosen as the active detector elements. Three identical modules, each of size $16 \mathrm{~m} \times 16 \mathrm{~m} \times 14.5 \mathrm{~m}$, will be constructed.

RPCs consist of two parallel plate electrodes of high bulk resistivity $\left(\sim 10^{12} \Omega \mathrm{cm}\right)$ which provide a constant and uniform electric field in the gap between them when a high voltage $(\sim 10 \mathrm{kV})$ is applied across them. The gap contains a gaseous ionizing medium which is a mixture of R134a $\left(\mathrm{C}_{2} \mathrm{H}_{2} \mathrm{~F}_{4}, 95 \%\right)$, Isobutane $\left(\mathrm{iC}_{4} \mathrm{H}_{10}, 4.5 \%\right)$ and Sulphur hexafluoride $\left(\mathrm{SF}_{6}, 0.5 \%\right)$. Freon acts as a secondary electron quencher with high probability for primary ionization and Isobutane acts as an absorber of UV photons. $\mathrm{SF}_{6}$ is required to control the excess number of electrons in avalanche mode [5]. An incoming charged particle ionizes the gas medium followed by the propagation and multiplication of the $e^{-}$-ion pairs, which in turn induces a current signal on external pickup strips [6]. The variations in the gas mixture compositions significantly affects the signal and hence the performance of RPCs. The effect of variation of $i C_{4} H_{10}$ or $S F_{6}$ on the charge distribution and time resolution of the RPC were studied. 


\section{Experimental setup}

Two glass plates of thickness $3 \mathrm{~mm}$ manufactured by Saint-Gobain were cut in $30 \mathrm{~cm} \times 30 \mathrm{~cm}$ dimensions, pasted with a conductive tape (T-9149) of uniform surface resistance on the outer surfaces, and then used to fabricate an RPC of $2 \mathrm{~mm}$ gap. The RPC was operated at $10.4 \mathrm{kV}$ in avalanche mode with a mixture of three gases, viz. R134a $\left(\mathrm{C}_{2} \mathrm{H}_{2} \mathrm{~F}_{4}\right)$, Isobutane $\left(\mathrm{iC}_{4} \mathrm{H}_{10}\right)$ and Sulphur hexafluoride $\left(S F_{6}\right)$. The RPC was sandwiched between two pick-up panels comprising of 10 copper strips each of width $28 \mathrm{~mm}$, separated by a gap of $2 \mathrm{~mm}$. The middle strip was read out to study the performance of RPC.

Figure 1 shows the schematic of experimental setup to study the performance of the RPC. A cosmic muon telescope was made using three plastic scintillator paddles $\mathrm{P}_{1}, \mathrm{P}_{2}$ and $\mathrm{P}_{3}$ that are arranged vertically one above the other to get a 3-fold coincidence. The dimensions of $\mathrm{P}_{1}, \mathrm{P}_{2}$ and $\mathrm{P}_{3}$ in length $\times$ width $\times$ thickness are $30 \times 2 \times 1 \mathrm{~cm}^{3}, 30 \times 3 \times 1 \mathrm{~cm}^{3}$ and $30 \times 5 \times 1 \mathrm{~cm}^{3}$ respectively. The RPC was placed in such a way that the central strip was aligned with the telescope window defined by the paddles. A high voltage was applied across the electrodes of the RPC. The analog signals from the RPC were amplified with a preamplifier, since their amplitude is small in the avalanche mode of operation. The signals from the scintillator paddles and RPC were fed to the Data Acquisition System (DAQ). Scintillator paddle signals were converted to logic signals by a discriminator and ANDed in a logic unit to get the trigger pulse. The amplified RPC analog signal was connected to a linear FAN IN/FAN OUT (FIFO) to get two buffered output signals. One output signal was connected to an oscilloscope to measure the charge and time of the signal, and the other was converted to a logic signal by feeding it to a discriminator with a threshold voltage of $-20 \mathrm{mV}$.

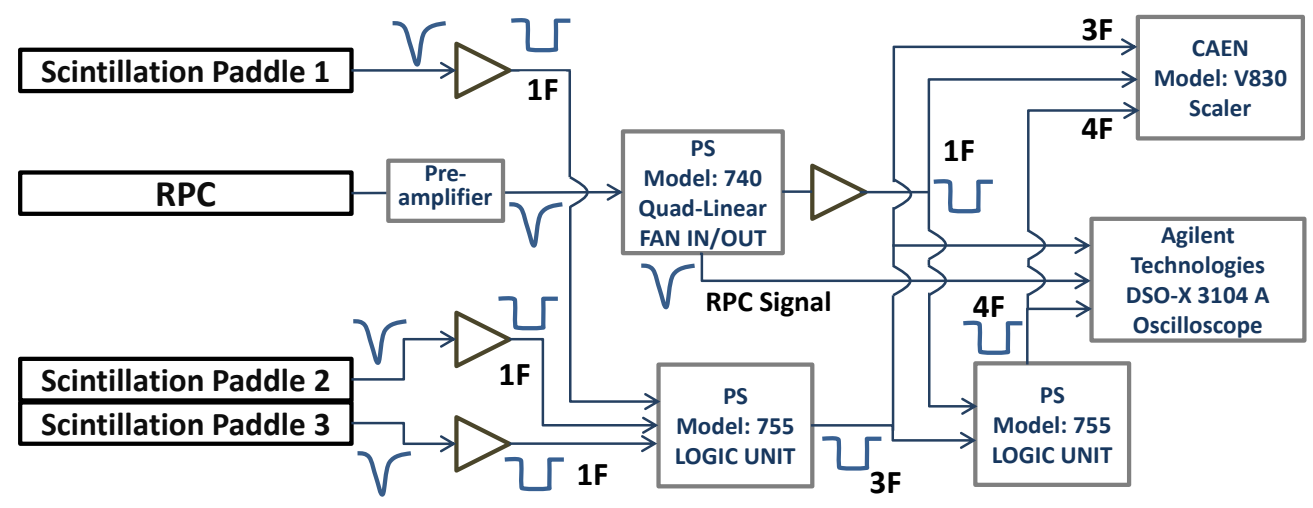

Figure 1: Schematic of experimental setup.

\subsection{Calibration of the gas system}

Mixing of the gases in different proportions and their supply to the RPC are executed by an online gas mixing and multi-channel distribution system developed by INO team [5]. Mass Flow Controllers (MFCs) are used to control the flow of each gas at its required rate. Therefore, the MFCs were calibrated according to the required flow of the gases in the RPCs. All the MFCs were calibrated by measuring the time taken to reduce the water level in an inverted burette (water downward displacement method). The calibration plots of MFCs corresponding to Freon, Isoutane and $\mathrm{SF}_{6}$ are shown in figure 2. The required proportion of three individual gases in the mixture 
were determined, and the flow rates were adjusted in the corresponding MFCs according to the calibration.
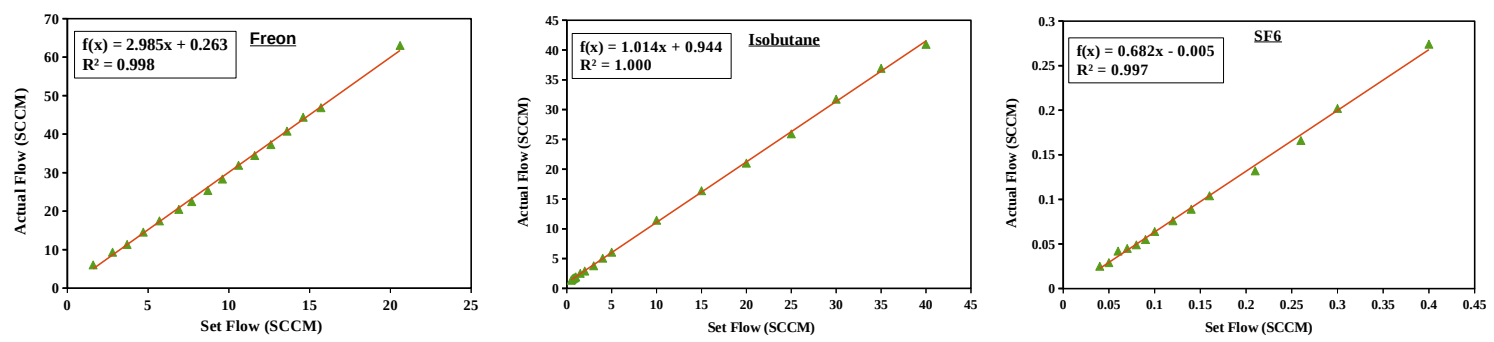

Figure 2: Calibration plots of MFCs corresponding to Freon, Isoutane and $\mathrm{SF}_{6}$.

\section{Performance of RPC at different percentages of $\mathrm{SF}_{6}$}

$\mathrm{SF}_{6}$ is a strong electronegative gas that captures free electrons. Therefore, addition of small amounts of $\mathrm{SF}_{6}(\sim 0.3 \%)$ significantly reduces the average pulse charge and suppresses the production of streamer signal in the RPCs [7]. To study the effect of variation of $\mathrm{SF}_{6}$ concentration, we have flown the $\mathrm{SF}_{6}$ at different proportions (from $0.3 \%$ to $0.8 \%$ in steps of $0.1 \%$ ) fixing the proportion of $i C_{4} H_{10}$ at $15 \%$. The charge induced on the copper strip was measured using an oscilloscope (DSO-X 3104 A) which stored the digitized analog pulses. Around 1000 signals were collected for each proportion of $\mathrm{SF}_{6}$, and their charge distributions and time resolutions were studied.

\subsection{Charge distribution}
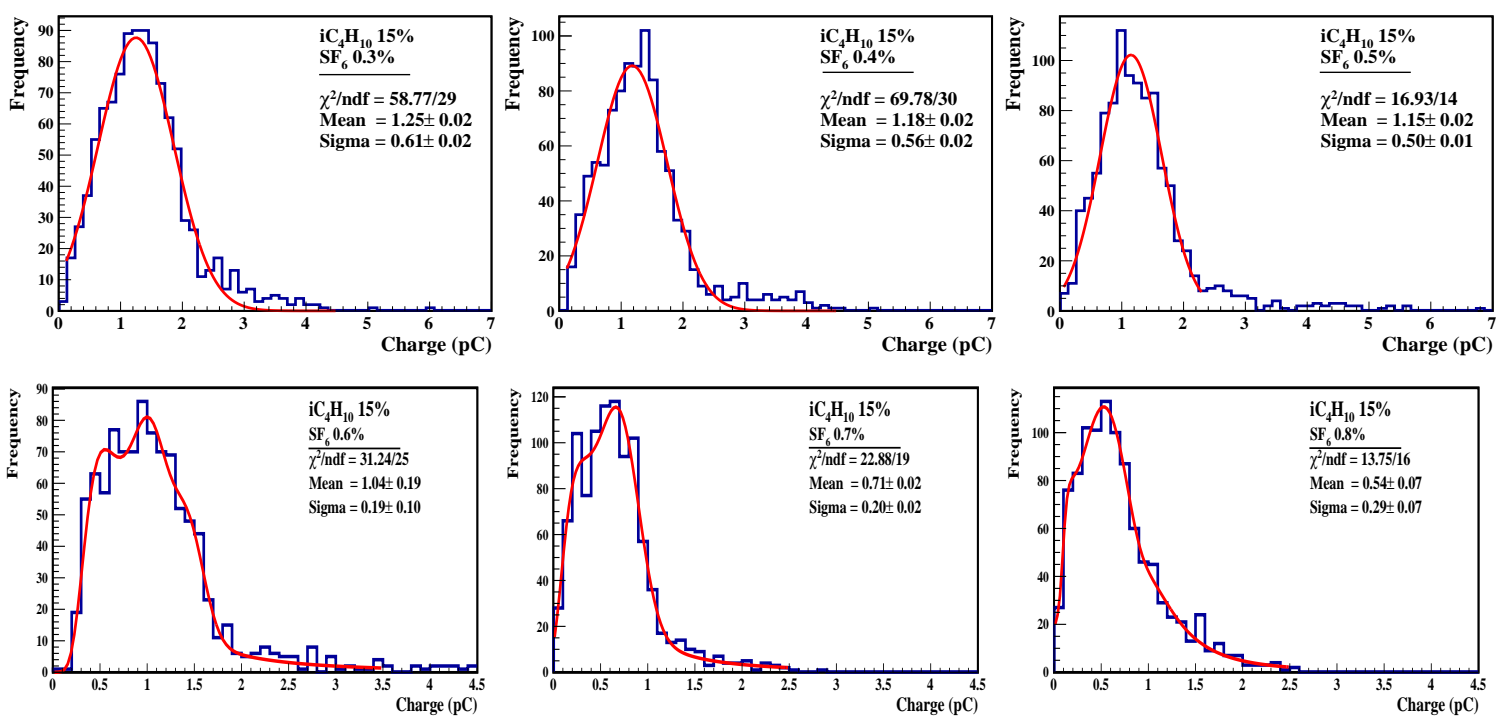

Figure 3: Charge distribution of the RPCs for various $\mathrm{SF}_{6}$ concentrations at same operating voltage.

The charge distributions of the avalanche signals collected at same operating voltage for various proportions of $\mathrm{SF}_{6}$ were fitted with Gaussian's and Landau functions as shown in figure 3. In general we have used Landau function for the rising part of the distribution (0-0.5). This Landau function convoluted with the Gaussian which is used for peak as well as tail part of the distribution. Mean 
and sigma collected from the combined distribution function. As the concentration of $\mathrm{SF}_{6}$ increases from $0.3 \%$ to $0.8 \%$, the charge spectra gets narrower and the peak is shifted to the left side. This indicates that only a lesser number of events induces an adequate amount of charge in the strip at higher concentration of $\mathrm{SF}_{6}$. Thus the mean of the charge distribution decreases with an increase in the $\mathrm{SF}_{6}$ concentration as shown in the figure 4a. This behaviour is due to the capture of the electrons in the gas gap by $\mathrm{SF}_{6}$ because of its high electron affinity $(1.05 \pm 0.10 \mathrm{eV})[7,8]$. This reduces the multiplication of electrons in the gas gap. Therefore, the muon events, which cause the production of very less number of primary charge pairs in the gap, would not be able to induce signals above threshold on read-out electrodes on that particular applied high voltage. This results the displacement of working point towards higher field intensity which could increase the noise signals The efficiency on the working point at $10.4 \mathrm{kV}$ is shown in the figure $4 \mathrm{~b}$ which reduced by the addition of $\mathrm{SF}_{6}$ in higher proportions.

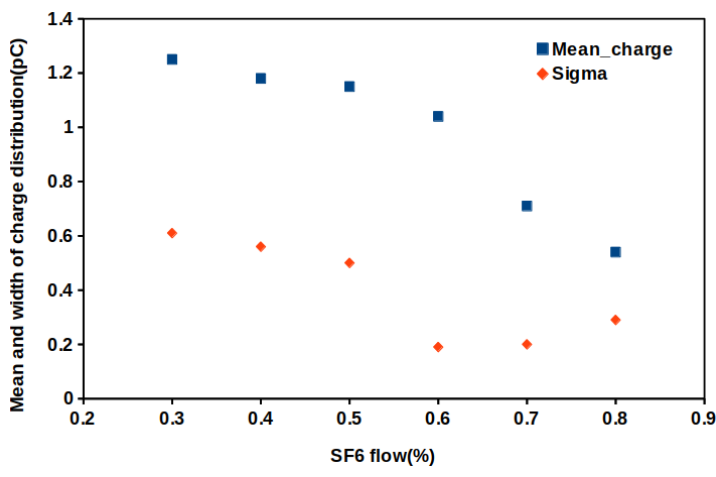

(a)

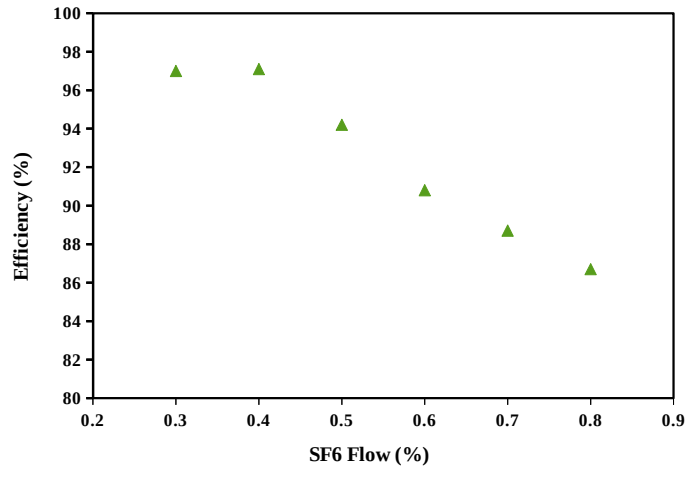

(b)

Figure 4: (a) The mean and width of charge distributions. (b) The efficiency of RPC. Both were plotted as a function of $\mathrm{SF}_{6}$ concentration.

\subsection{Time resolution}

The time distribution of the signals collected with RPC at different $\mathrm{SF}_{6}$ proportions is shown in figure 5. There was no significant effect on the time resolution of the RPCs by the variation in $\mathrm{SF}_{6}$ concentration. 

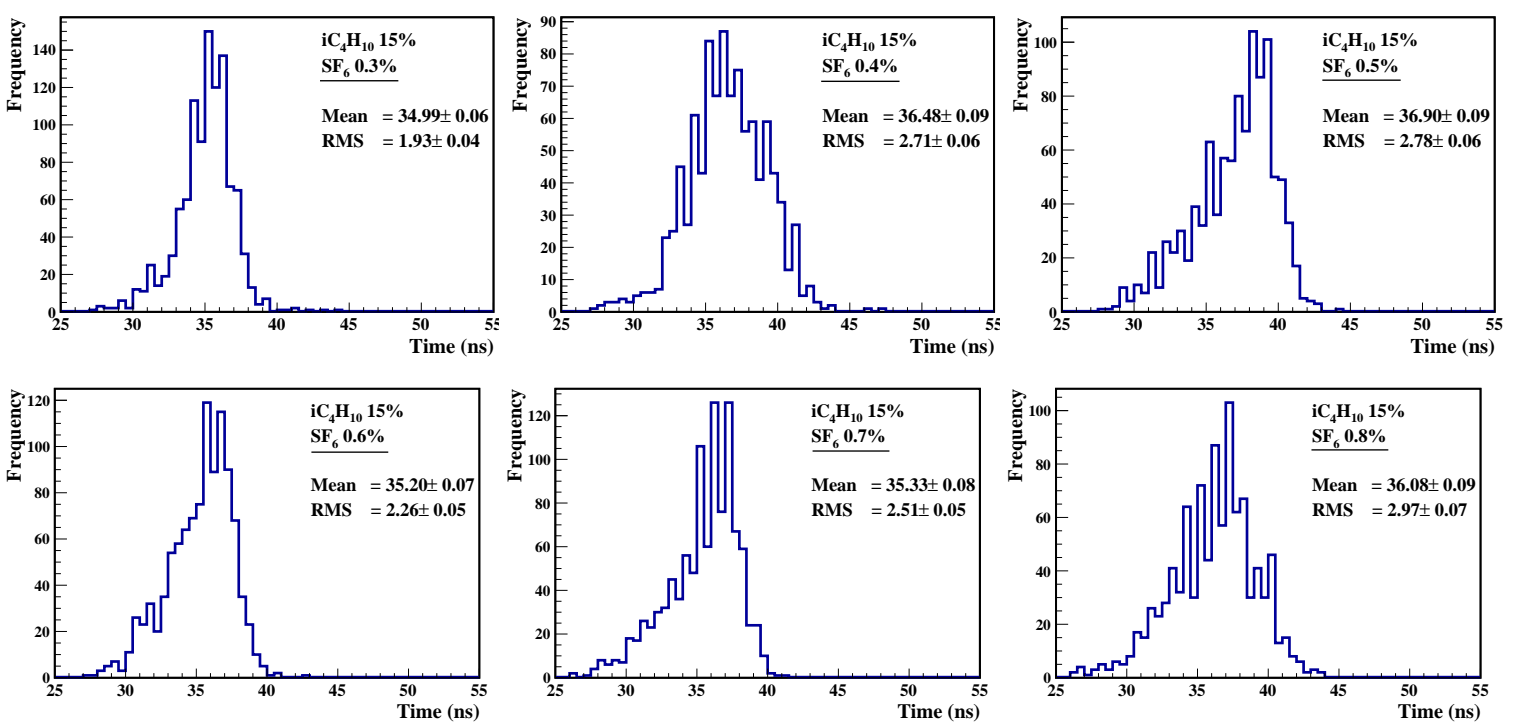

Figure 5: Time distribution of the RPCs for various $\mathrm{SF}_{6}$ concentrations at same operating voltage.

\section{Performance of $\mathrm{RPC}$ at different percentages of Isobutane $\left(\mathrm{iC}_{4} \mathrm{H}_{10}\right)$}

Isobutane molecules act as quenchers by absorbing UV photons in a wide range of energies and thereby reducing the formation of secondary avalanches. The performance of RPC was studied at various proportions of Isobutane keeping $\mathrm{SF}_{6}$ at $0.4 \%$. The charge distributions and time resolutions were measured from 1000 signals collected for each proportion of Isobutane. The dependance of average signal charge and time on the Isobutane concentration is analysed.

\subsection{Charge distribution}

The charge distributions of the signals collected at various Isobutane proportions were fitted with Gaussians and are shown in figure 6. It is observed from the plot that the RPC is less sensitive to the variations in the proportion of Isobutane in comparison with its sensitivity to $\mathrm{SF}_{6}$ variation. The plot of efficiency versus Isobutane concentration, shown in figure 7, implies that the change in the Isobutane concentration by $10 \%$ reduces the efficiency by only $2 \%$ approximately.

\subsection{Time resolution}

The time distribution of the signals collected with RPC for various Isobutane proportions is shown in figure 8 . The time resolution of the RPC is found to be $2 \mathrm{~ns}$ approximately. The time resolution is not affected significantly by the variation in Isobutane concentration.

\section{Conclusion}

The variations in the compositions of gaseous ionizing medium in the RPC affects the propagation and multiplication of charge carriers and hence the performance of the RPCs. Therefore, the performance of RPC was studied at different concentration of $\mathrm{SF}_{6}$ and Isobutane. A slight increase in the $\mathrm{SF}_{6}$ concentration causes significant reduction in the mean of the charge induced on the strips as well as in the efficiency. This is due to the capture of the electrons in the gas gap by $\mathrm{SF}_{6}$ because of its high electron affinity. However, RPC is less sensitive for charge collection to the variations 

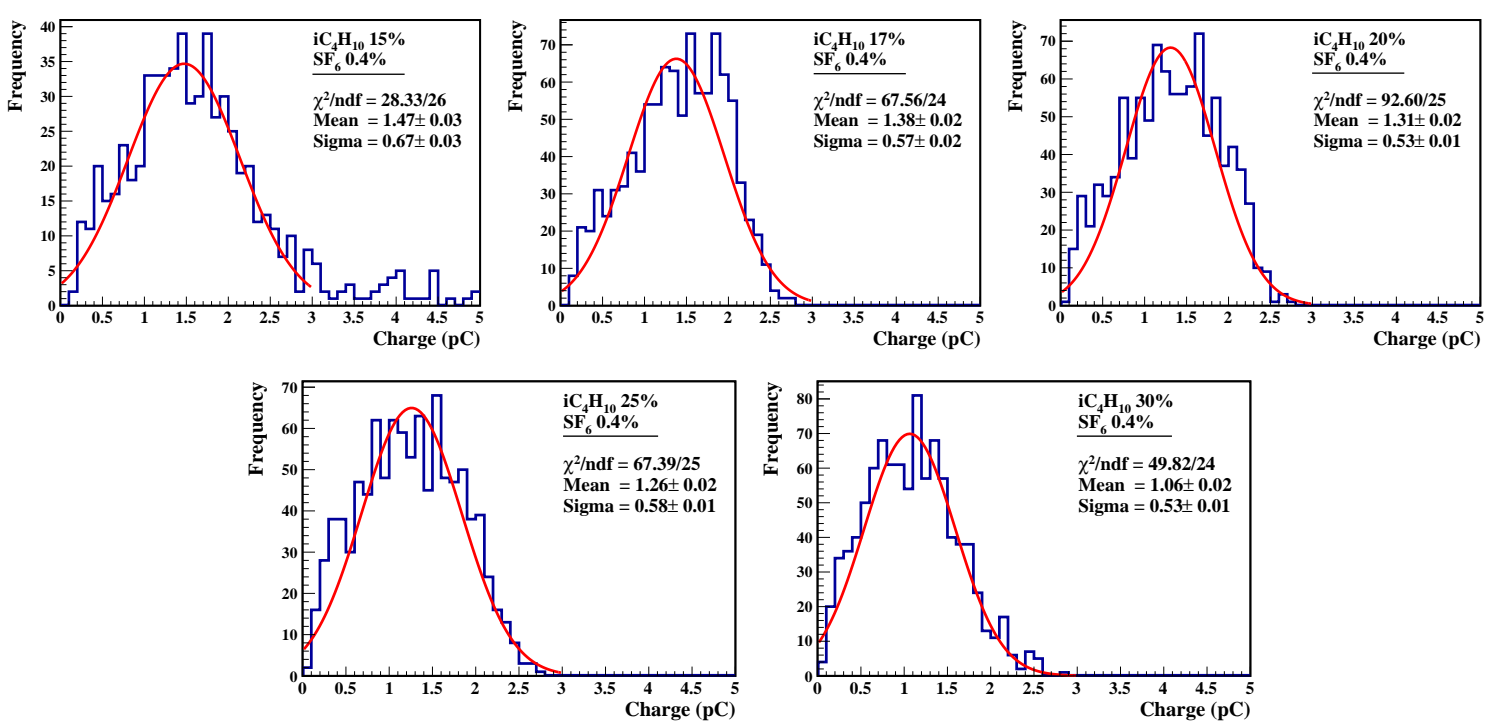

Figure 6: Charge distribution of the RPCs different Isobutane concentrations at same operating voltage.

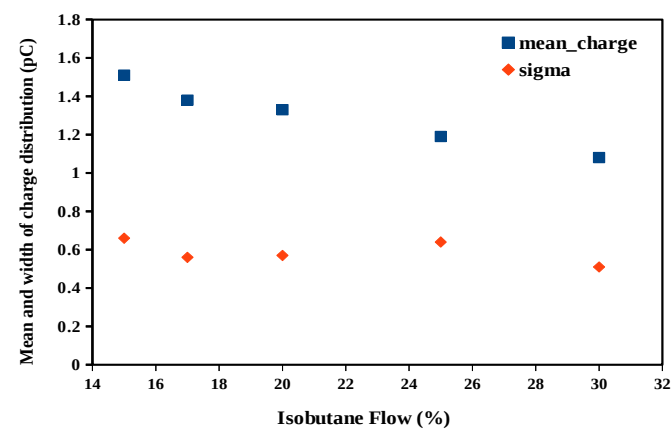

(a)

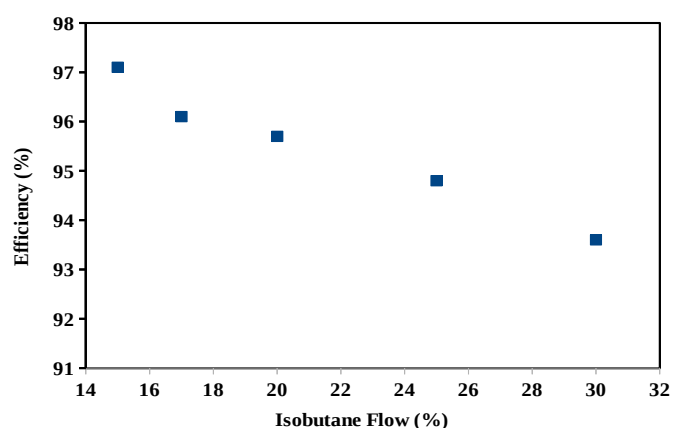

(b)

Figure 7: (a) The mean and width of charge distributions. (b) The efficiency of RPC. Both were plotted as a function of Isobutane concentration.

in the concentration of Isobutane compare to the variation of $\mathrm{SF}_{6}$. The mean charge and efficiency were observed to be varied slightly by increasing the concentration of Isobutane gas. This is due to the fact that Isobutane absorbs the UV photons, and hence controls the formation of secondary avalanches. There was no significant difference in the time resolution of RPC by the variation in the concentration of $\mathrm{SF}_{6}$ or Isobutane.

\section{Acknowledgements}

This work was done with the support of the Department of Atomic Energy (DAE), and the Department of Science and Technology (DST), Government of India. The authors would like to thank INO collaboration for their help throughout the work. 

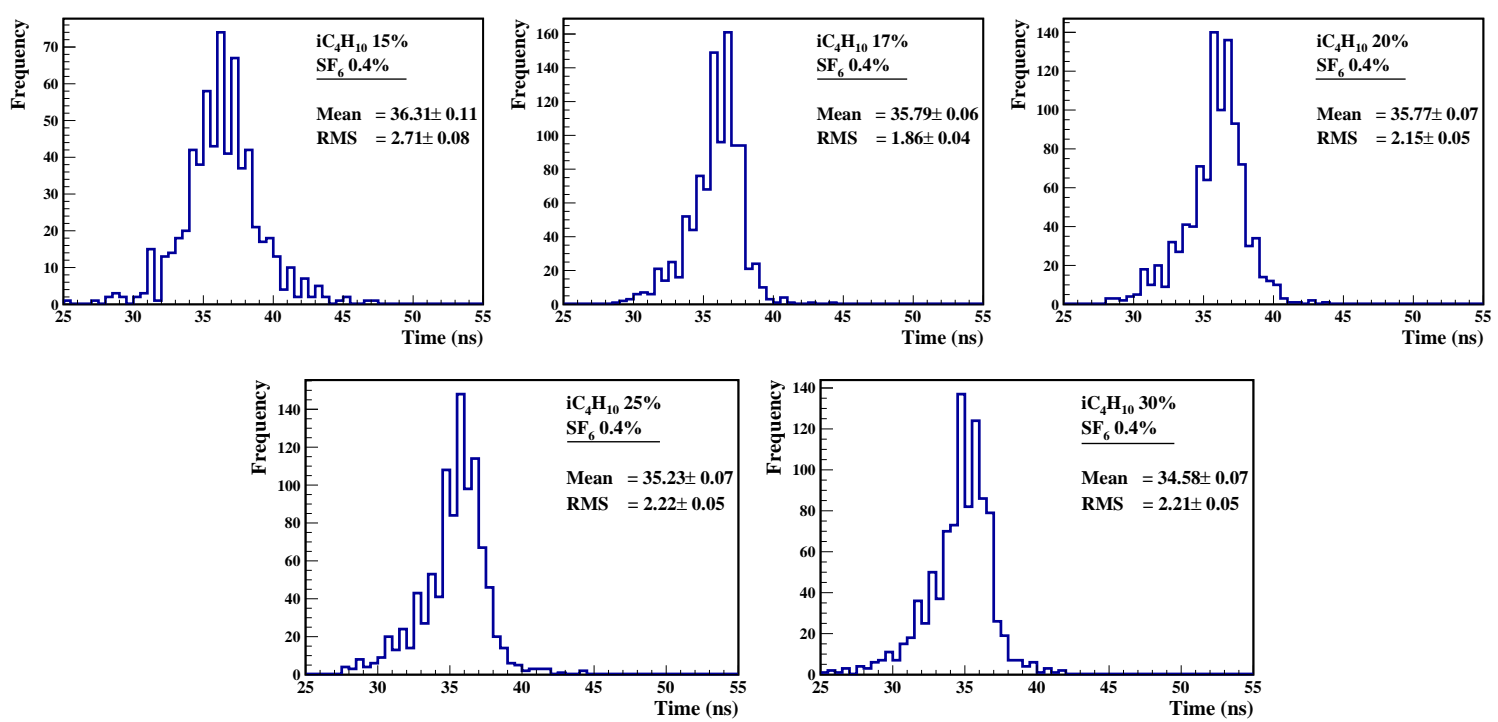

Figure 8: Charge distribution of the RPCs different Isobutane concentrations at same operating voltage.

\section{References}

[1] R. Santonico, New generations of RPCs for accelerator and non-accelerator physics, JINST 8 (2013) P04023.

[2] D. Watts, G. Borghi, F. Sauli, U. Amaldi, The use of multi-gap resistive plate chambers for in-beam PET in proton and carbon ion therapy, Journal of Radiation Research 54 (2013) 136-142.

[3] R. Santonico and R. Cardarelli, Development of resistive plate counters, Nucl. Instr. and Meth. A 187 (1981) 377-380.

[4] S. Ahmed et al., Invited review: Physics potential of the ICAL detector at the India-based Neutrino Observatory (INO), Pramana 88 (2017) 79.

[5] S.D. Kalmani et al., On-line gas mixing and multi-channel distribution system, Nucl. Instrum. Meth. A 602 (2009) 845-849.

[6] M. Abbrescia et al., The simulation of resistive plate chambers in avalanche mode: charge spectra and efficiency, Nucl. Instr. and Meth. A 431 (1999) 413-427.

[7] P. Camarri et al., Streamer suppression with $S_{6}$ in RPCs operated in avalanche mode, Nucl. Instrum. Meth. A 414 (1998) 317-324.

[8] M. Salim et al., Experimental and numerical studies on the effect of $S F_{6}$ in a glass RPC, JINST 7 (2012) P11019. 